\title{
Despopulação neuronal pancreática em chagásicos crônicos
}

\author{
Neuronal pancreatic depopulation in chronic \\ Chagas' disease patients
}

\author{
Ademir Rocha, Luiz Carlos Marques de Oliveira, \\ Renato Silva Alves e Edison Reis Lopes
}

\begin{abstract}
Resumo Revendo a literatura não encontramos estudos anatômicos dos gânglios intrapancreáticos na forma crônica da doença de Chagas; lesões dos mesmos poderiam explicar, ao menos em parte, os distúrbios funcionais do pâncreas exócrino e endócrino descritos nesta forma da doenca. Decidimos então analisar morfologicamente tais gânglios. Para isso, estudamos segmentos transversais da cabeça, corpo e cauda do pâncreas de doze chagásicos crônicos, com idade média de 46,5 \pm 9,1 anos, e quatorze controles, com idade média de 41,2 $\pm 11,0$ anos. Os segmentos foram processados histologicamente e seccionados de forma seriada até o esgotamento, analisando-se os cortes múltiplos de sete. Para análise estatística, usamos o teste não-paramétrico de Mann-Whitney. Na cabeça do pâncreas, a contagem de neurônios teve média de 57,3 \pm 50,8 para o grupo chagásico e 117,5 \pm 99,0 para o grupo controle $(p<0,05)$; no corpo, $25,9 \pm 19,4$ para o grupo chagásico e $54,7 \pm 47,8$ para o controle $(p<0,05)$; na cauda, $23,4 \pm 16,3$ para o chagásico e 54,1 $\pm 29,2$ para o controle $(p<0,01)$, sendo a contagem total de 106,6 $\pm 71,1$ para o chagásico e 226,3 $\pm 156,5$ para o controle $(p<0,01)$. Nossos achados nos permitiram concluir que: a) ocorreu despopulação neuronal estatisticamente significante no grupo chagásico em relação ao controle, em cada segmento pancreático analisado, bem como no órgão como um todo; b) $50 \%$ dos chagásicos tiveram número total de neurônios inferior ao menor número dos controles (80); c) $75 \%$ e $91,6 \%$ dos chagásicos tiveram número de neurônios inferior, respectivamente, à mediana (171) e à média (226) do grupo controle; d) assim, a despopulacão neuronal pancreática foi frequente, porém não constante; e) o fator idade não pareceu ter sido o responsável pela despopulação neuronal dos chagásicos.
\end{abstract}

Palavras-chaves: Doença de Chagas. Gânglios intrapancreáticos. Inervação pancreática. Tripanossomíase sul-americana.

\begin{abstract}
We have not found any anatomical studies about the intrapancreatic ganglia in the chronic Chagas' disease. The lesions in these structures could explain at least in part the functional disturbances in the exocrine and endocrine pancreas described in this form of the disease. Thus we decided to morphologically analyze these ganglia. For this analysis, we studied transversal segments of the head, body and tail of the pancreas of twelve chronic chagasics whose mean age were 46,5 \pm 9,1 years and fourteen controls, mean age 41,2 \pm 11,0 years. These segments were histologically processed and cut into sections in a seriate form up to the end and one cut of each seven was analyzed. For statistical analysis we used the non-parametric test of Mann-Whitney. In the head of the pancreas, the mean count of neurons was 57,3 $\pm 50,8$ in the chagasic group and 117,5 $\pm 99,0$ for the control group ( $p<0,05)$; in the body 25,9 $\pm 19,4$ for the chagasic group and 54,7 \pm 47,8 for the control group $(p<0,05)$; in the tail 23,4 $\pm 16,3$ for the chagasic group and 54,1 $\pm 29,2$ for the control group ( $p<0,01)$, the total count being 106,6 $\pm 71,1$ for the chagasic group and 226,3 $\pm 156,5$ for the controls ( $p<0,01)$. Our data permitted us to conclude that: a) there was a statistically significant neuronal depopulation in the chagasic group, as compared to the control group, in each pancreatic segment that was analyzed, as well as in the organ as a whole; b) $50 \%$ of the cagasics had the total number of neurons smaller than the lowest number observed in the controls (80); c) $75 \%$ and $91.6 \%$ of the chagasics had the number of neurons smaller than, respectively, the median (171) and the mean (226) of the control group; d) therefore, the pancreatic neuronal depopulation was common, but not constant; e) the variable age was apparently not responsible for the neuronal depopulation of the chagasics.
\end{abstract}

Key-words: Chagas' disease. Intrapancreatic ganglia. Pancreatic innervation. South-American trypanosomiasis.

Serviço de Anatomia Patológica do Hospital de Clínicas da Universidade Federal de Uberlândia, Uberlândia, MG. Endereço para correspondência: Dr. Ademir Rocha. Av. José Zacharias Junqueira 282/102, 38400-112 Uberlândia, MG Recebido para publicação em 09/05/97. 
As alterações morfológicas do tubo digestivo na doença de Chagas humana e experimental têm sido exaustivamente descritas 417182131 . Há poucos relatos, no entanto, em relação a possíveis lesões pancreáticas, seja no homem6 192932 , seja em animais de experimentação5 714152426 .

As principais alterações morfológicas detectadas nos diferentes segmentos do tubo digestivo residem nos plexos nervosos intrínsecos, consistindo em redução numérica dos neurônios, ganglionite, periganglionite, etc; a desnervação seria fator essencial na gênese dos distúrbios motores que freqüentemente são verificados no esôfago e no intestino grosso de chagásicos crônicos 182131.

Em relação aos órgãos anexos do tubo digestivo, diminuição do número de neurônios também já foi caracterizada ao nível da vesícula biliar 9, mas não nos consta que haja estudos anatômicos da inervação das glândulas salivares e do pâncreas de chagásicos crônicos.

Lesões da inervação intrínseca pancreática poderiam explicar, ao menos em parte, distúrbios metabólicos relacionados à função exócrina23 e endócrina do órgão8 2527283334 descritos na forma crônica da doença de Chagas.

Diante do exposto, decidimos analisar morfologicamente os gânglios intrapancreáticos em chagásicos crônicos, sob os aspectos quantitativo (número de neurônios totais e por segmento pancreático) e qualitativo (ganglionite, periganglionite, alterações regressivas neuronais, etc).

\section{MATERIAL E MÉTODOS}

O material de estudo constou de doze pâncreas de chagásicos crônicos (dez com a forma cardíaca e dois com a cárdio-digestiva da doença) e quatorze de não-chagásicos (controles), obtidos à necropsia do Serviço de Anatomia Patológica do Hospital de Clínicas da Universidade Federal de Uberlândia, MG e no Posto MédicoLegal da mesma cidade. Nas Tabelas 1 e 2, apresentamos os dados relativos ao sexo, idade e peso do pâncreas de cada um dos casos dos grupos chagásico e controle, respectivamente; a Tabela 1 ainda contém a forma clínica de cada caso de doença de Chagas. Não houve diferença entre os dois grupos em relação à idade e ao peso do pâncreas. O diagnóstico de infecção chagásica baseou-se na positividade das reações sorológicas para Trypanosoma cruzi no líquido pericárdico e/ou sangue (fixação do complemento, hemaglutinação, ELISA, imunofluorescência) e nos achados anátomopatológicos macro e microscópicos. Os controles eram indivíduos aparentemente sãos, com sorologia negativa para Trypanosoma cruzi, e que tiveram morte violenta (vítimas de homicídio, acidente de trânsito, etc.). Excluíramse casos com história pregressa de alcoolismo crônico ou "diabetes mellitus", bem como aqueles em que o exame necroscópico evidenciou pancreatite.

Os pâncreas eram dissecados, pesados e parcialmente seccionados no sentido longitudinal; a secção destinava-se a facilitar a fixação. Esta

\begin{tabular}{|c|c|c|c|c|}
\hline Caso & Sexo & $\begin{array}{l}\text { Idade } \\
\text { (anos) }\end{array}$ & $\begin{array}{c}\text { Forma da } \\
\text { doença }\end{array}$ & $\begin{array}{c}\text { Peso do } \\
\text { pâncreas (g) }\end{array}$ \\
\hline 1 & $\mathrm{M}$ & 38 & cardlaca & 80 \\
\hline 2 & $\mathrm{~F}$ & 36 & cardlaca & 93 \\
\hline 3 & $\mathrm{~F}$ & 48 & cardlaca & 80 \\
\hline 4 & $F$ & 47 & cardlaca & 85 \\
\hline 5 & $F$ & 47 & cardlaca & 105 \\
\hline 6 & $M$ & 55 & cardlaca & 115 \\
\hline 7 & M & 55 & cardlaca & 150 \\
\hline 8 & M & 38 & cardlaca & 103 \\
\hline 9 & $M$ & 43 & cárdio-digestiva & 100 \\
\hline 10 & $M$ & 65 & cárdio-digestiva & 47 \\
\hline 11 & $M$ & 51 & cardlaca & 116 \\
\hline 12 & $\mathrm{~F}$ & 35 & cardlaca & 79 \\
\hline $\mathrm{X} \pm \mathrm{DP}$ & & $46,5 \pm 9,1$ & & $96,1 \pm 25,5$ \\
\hline
\end{tabular}


Tabela 2 - Dados relativos ao grupo controle.

\begin{tabular}{cccc}
\hline Caso & Sexo & $\begin{array}{c}\text { Idade } \\
\text { (anos) }\end{array}$ & $\begin{array}{c}\text { Peso do } \\
\text { pâncreas }(\mathrm{g})\end{array}$ \\
\hline 1 & $\mathrm{M}$ & 54 & 110 \\
2 & $\mathrm{M}$ & 29 & $\mathrm{NP}$ \\
3 & $\mathrm{~F}$ & 44 & 100 \\
4 & $\mathrm{~F}$ & 48 & 109 \\
5 & $\mathrm{M}$ & 19 & 100 \\
6 & $\mathrm{M}$ & 37 & 150 \\
7 & $\mathrm{M}$ & 45 & 100 \\
8 & $\mathrm{M}$ & 43 & 165 \\
9 & $\mathrm{~F}$ & 30 & 85 \\
10 & $\mathrm{~F}$ & 32 & 110 \\
11 & $\mathrm{~F}$ & 50 & 110 \\
12 & $\mathrm{~F}$ & 51 & 100 \\
13 & $\mathrm{M}$ & 37 & 100 \\
14 & $\mathrm{M}$ & 58 & 125 \\
\hline X \pm DP & & $41,2 \pm 11,0$ & $112,6 \pm 22,1$ \\
\hline
\end{tabular}

$\mathrm{M}=$ masculino; $\mathrm{F}=$ feminino; $\mathrm{NP}=$ não pesado; $\mathrm{X}=$ média; $\mathrm{DP}=$ desvio padrão

era feita por imersão em formol a $10 \%$ por tempo variável, não inferior a 24 horas. A seguir, retiravam-se três segmentos transversais de aproximadamente $0,5 \mathrm{~cm}$ de espessura, ao nível das partes médias da cabeça, corpo e cauda; a largura dos segmentos correspondia à das regiões do pâncreas nos locais de retirada. O material era, então, processado histologicamente para inclusão em parafina.

Com o objetivo de contar os neurônios intrínsecos, os três blocos contendo os segmentos incluídos eram cortados seriadamente, até o esgotamento, com espessura de 7 micrômetros. Para evitar a contagem repetida de um mesmo neurônio, montamos apenas cortes múltiplos de sete, de acordo com técnica de Köberle17. Os cortes para a contagem neuronal eram corados por hematoxilina-eosina (HE). Cortes intermediários esparsos de todos os blocos eram montados para eventuais colorações especiais. Os preparados histológicos eram analisados de forma qualitativa, após o que se contavam os neurônios, utilizando-se ocular de 10x e objetiva de 10x. Em cada caso, estudaram-se cerca de 60 a 70 preparados histológicos (20 a 24 por segmento).

Para análise dos resultados, utilizamos teste não paramétrico (Mann-Whitney) levando-se em consideração a natureza das distribuições dos valores da variável estudada. Para as comparações das médias etárias e dos pesos médios dos pâncreas, empregamos o teste "t" de Student.

\section{RESULTADOS}

A Tabela 3 mostra as médias, os desviospadrão e as medianas dos números de neurônios em cada segmento pancreático analisado. Os

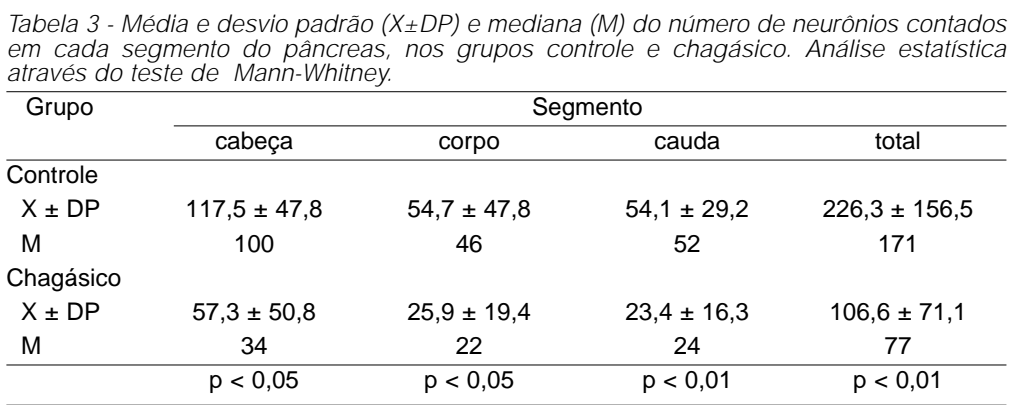


resultados mostram que em todos os segmentos (cabeça, corpo e cauda), assim como na contagem total dos três segmentos, o número de neurônios foi significativamente menor no grupo chagásico em relação ao grupo controle. $\mathrm{Na}$ Tabela 4, mostramos, individualmente, a contagem total de neurônios pancreáticos nos cortes examinados, em ambos os grupos. No grupo chagásico, com 12 indivíduos, esta contagem variou de 39 a 239, com média de 106,6 e desvio-padrão de 71,1. No grupo controle, a variação foi de 80 a 651 neurônios, com média de 226,3 e desvio padrão de 156,5. Esta Tabela dá a noção da diferença entre os dois grupos avaliados. Não encontramos ganglionite nos cortes analisados, e somente em um caso verificamos um único foco de periganglionite.

Tabela 4 - Contagem total de neurônios pancreáticos em cada caso dos grupos chagásico e controle

\begin{tabular}{ccc}
\hline Caso & Chagásico & Controle \\
\hline 1 & 39 & 80 \\
2 & 39 & 160 \\
3 & 239 & 125 \\
4 & 52 & 167 \\
5 & 40 & 223 \\
6 & 72 & 101 \\
7 & 47 & 174 \\
8 & 148 & 651 \\
9 & 174 & 265 \\
10 & 195 & 127 \\
11 & 82 & 100 \\
12 & 153 & 289 \\
13 & & 263 \\
14 & & 444
\end{tabular}

\section{DISCUSSÃO}

A inervação pancreática abrange fibras autônomas (simpáticas e parassimpáticas) e sensitivas; as fibras parassimpáticas têm origem vagal; as simpáticas, do tronco esplâncnico. O sistema nervoso autônomo intrapancreático é representado por nervos e gânglios. Os textos em geral se referem superficialmente aos gânglios; segundo Harris 16 são parassimpáticos e situam-se especialmente ao nível do estroma interlobular, parecendo concentrar-se nas regiões da cabeça e do colo pancreáticos. Refere-se a existência de nervos peptidérgicos associados aos gânglios intrapancreáticos29. É preciso frisar que há muitas lacunas a preencher em relação aos gânglios intrínsecos (distribuição, morfologia, alterações com o envelhecimento, etc).

A secreção exócrina do pâncreas depende da integração de estímulos nervosos (especialmente parassimpáticos) e hormonais (gastrina, secretina, colecistocinina). Por outro lado, a regulação da secreção das ilhotas de Langerhans envolve, além do substrato, os gânglios pancreáticos, fibras nervosas colinérgicas, noradrenérgicas e peptidérgicas; haveria ainda um controle interno abrangendo insulina, glucagon e somatostatina22. Os gânglios intrínsecos podem exercer um papel de "marca-passo" na regulação da secreção de insulina, pelo menos em cães 30 .

Distúrbios metabólicos diversos detectados em chagásicos crônicos, relacionados às funções exócrina e endócrina do pâncreas, poderiam envolver lesões de sua inervação autonômica. Com freqüência, levanta-se a suspeita de que tais lesões comprometem, essencialmente, o chamado eixo êntero-pancreático, do qual participam fibras vagais provenientes do duodeno23. Não se tem questionado, todavia, a hipótese de que os distúrbios funcionais do pâncreas se devam a alterações dos gânglios intrapancreáticos. Propusemo-nos a investigar a possibilidade de tais lesões considerando que: 1) existem íntimas relações anatômicas, funcionais e embriológicas entre o tubo digestivo e o pâncreas, e 2) na doença de Chagas crônica, acha-se muito bem caracterizado o dano ganglionar ao longo de todos os segmentos do tubo digestivo, traduzido por perda neuronal, fenômenos regressivos dos neurônios, ganglionite, etc'123101112131718.

Revendo a escassa literatura sobre lesões pancreáticas na fase crônica da doença de Chagas humana, não localizamos qualquer estudo que avaliasse sistematicamente os gânglios do sistema nervoso intrapancreático. $\mathrm{Na}$ falta de um método previamente descrito para tal finalidade, adaptamos os utilizados por Köberle e seus discípulos e colaboradores na avaliação dos plexos de Meissner e Auerbach do tubo digestivo3 101112131718 . A metodologia adaptada nos pareceu fornecer dados plenamente confiáveis.

No presente estudo, como mostram as Tabelas 3 e 4, foi frequente a despopulação neuronal pancreática entre os chagásicos; de fato, em apenas três dos doze casos analisados constatou-se número total de neurônios acima 
da mediana do grupo de controle (171 neurônios), e somente 1/12 mostrou total de neurônios acima da média de controles $(226,3)$; além do mais, seis dos doze chagásicos tiveram neurônios totais abaixo da menor quantidade verificada no grupo controle (80 neurônios). Globalmente, o grupo chagásico teve um valor médio de neurônios totais significativamente inferior ao grupo controle; este achado assemelha-se aos já constatados nos diferentes segmentos do tubo digestivo. Por outro lado, houve chagásicos com número total de neurônios comparável ou mesmo superior ao de muitos controles; fato similar já foi verificado na avaliação quantitativa de neurônios no esôfago, no intestino grosso e no coração de chagásicos crônicos 121718 20. Isto sugere que a desnervação não representa um fato obrigatório na evolução da tripanosomíase americana, quer do pâncreas, quer noutros órgãos.

Também chamou a atenção, neste estudo, que a redução quantitativa dos neurônios entre os chagásicos foi significativa nos três segmentos pancreáticos analisados, indicando a ocorrência de despopulação neuronal difusa. Pareceu-nos ainda digna de nota a constatação, nos grupos chagásico e controle, de um número médio de neurônios maior na cabeça que no corpo e na cauda do órgão; realmente, a literatura destaca que os gânglios intrapancreáticos parecem concentrar-se nas regiões cefálica e do colo16.

Vale ainda comentar a ausência de ganglionite pancreática (e o achado de um único foco de periganglionite) nos cortes histológicos analisados. O fato poderia indicar que a despopulação neuronal do órgão teria ocorrido há muito tempo, até mesmo na fase aguda da tripanossomíase, não havendo fatores locais que the promovessem o agravamento. Curiosamente, Saldanha29 analisando o pâncreas de 17 chagásicos e oito controles falecidos em Uberaba, MG, cidade situada a cerca de $110 \mathrm{~km}$ de Uberlândia, referiu o encontro de infiltração leucocitária e fibrose, discretas ou moderadas, em gânglios "de alguns chagásicos crônicos". Tendo em conta que o autor se preocupou, nesse trabalho, em analisar de modo especial as ilhotas de Langerhans e não os gânglios pancreáticos, surpreendemonos com os dados referidos e não vislumbramos uma explicação clara para tal diferença entre seus achados e os nossos. No esôfago e no cólon de chagásicos crônicos, apesar de relativamente freqüentes, focos de ganglionite nem sempre são verificados, principalmente na ausência de "megas"; noutros segmentos do tubo, tais focos habitualmente não são referidos'12 3101112131718.

A desnervação pancreática nos chagásicos de nosso estudo não nos pareceu relacionada à idade dos pacientes, considerando que tal fator, em relação aos controles, não mostrou diferença significativa. Além disso, o indivíduo mais velho do grupo chagásico, com 65 anos, tinha número total de neurônios (195) maior que a mediana do grupo controle (171). Nossa casuística incluiu apenas dois chagásicos com "mega" (Tabela 1), nos quais contamos 174 e 195 neurônios. Assim, apesar de não termos dados suficientes para estabelecer inferências sobre possíveis diferenças de comportamento ganglionar entre as formas cardíaca e digestiva da doença, salientamos que parece não existir correlação obrigatória entre desnervação pancreática e a presença de "megas".

Deve-se ressaltar, por fim, que a constatação de desnervação pancreática no grupo chagásico naturalmente não exclui a possibilidade de lesão concomitante do chamado eixo ênteropancreático, aventada para justificar certos distúrbios metabólicos associados ao órgão23 25.

\section{REFERÊNCIAS BIBLIOGRÁFICAS}

1. Adad SJ. Contribuição ao estudo da anatomia patológica e da patogênese do megaesôfago chagásico. Tese de mestrado, Faculdade de Medicina do Triângulo Mineiro, Uberaba, MG, 1989.

2. Adad SJ. Contribuição ao estudo da anatomia patológica e da patogênese do megacólon chagásico. Tese de doutorado, Faculdade de Medicina do Triângulo Mineiro, Uberaba, MG, 1996.

3. Alcântara FG, Costa RB. Jejunopatia chagásica. Revista Brasileira de Medicina 23:316-317, 1966.

4. Andrade ZA, Andrade SG. Patologia. In: Brener Z, Andrade ZA (eds) Trypanosoma cruzi e doença de Chagas, Guanabara Koogan, Rio de Janeiro, p. 199247, 1979.

5. Bastos OMP, Costa SCG, Alencar AA, Bauer PG. Calcificação na musculatura esquelética em camundongos infectados com Trypanosoma cruzi. Revista Brasileira de Neurologia 22:117-122, 1986.

6. Brito-Costa R, Neto Jr EM, Martum J. Estudo sobre a secreção glandular exócrina do aparelho digestivo e dos brônquios na moléstia de Chagas humana. Revista do Instituto de Medicina Tropical de São Paulo 15:227-234, 1973.

7. Calabrese KS, Lagrange PH, Costa SCG. 
Trypanosoma cruzi: histopathology of endocrine system in immunocompromised mice. International Journal of Experimental Pathology 75:453-462, 1994.

8. Campos JO, Cançado JR. Curvas glicêmicas anormais observadas em pacientes com a forma crônica da moléstia da Chagas. O Hospital 62:275278, 1962.

9. Conte VP. Aspectos anátomo-funcionais da vesícula biliar em pacientes com megaesôfago chagásico. Revista do Hospital das Clínicas da Faculdade de Medicina da Universidade de São Paulo 36:69-77, 1981.

10. Costa RB, Alcântara FG. Gastropatia chagásica crônica. Revista Brasileira de Medicina 22:667-671, 1965.

11. Costa RB, Alcântara FG. Duodenopatia chagásica. Revista Brasileira de Medicina 23:158-166, 1966.

12. Costa RB, Alcântara FG. Plexos submucoso e mientérico do íleo humano na moléstia de Chagas. Revista Brasileira de Medicina 23:399-400, 1966.

13. Costa RB, Lima EC. Plexos submucoso e mientérico do cólon humano na moléstia de Chagas. Revista do Instituto de Medicina Tropical de São Paulo 6:211218, 1964.

14. Costa SCG, Calabrese KS, Alencar AA, Lagrange $\mathrm{PH}$. Trypanosoma cruzi invasion of structures related to development and central nervous system. Revista Brasileira de Neurologia 22:183-190, 1986.

15. Costa SCG, Lagrange PH, Hurtrel B, Kerr I, Alencar A. Role of $T$ lymphocytes in the resistance and immunopathology of experimental Chagas' disease. Annales d'Immunologie 135C:317-332, 1984.

16. Harris PF. Anatomy. In: Howat HT, Sarles H (eds) The exocrine pancreas. Saunders, London, UK, p.15-30, 1979.

17. Köberle F. Chagas' disease and Chagas' syndromes: the pathology of American trypanosomiasis. Advances in Parasitology 6:63-116, 1968.

18. Köberle F. Patogenia da moléstia de Chagas. In: Cançado JR (ed.) Doença de Chagas, Imprensa Oficial do Estado de Minas Gerais, Belo Horizonte, MG, p.238-260, 1968.

19. Lisbôa AC. Sôbre a forma congênita da doença de Chagas. Estudo anátomo-patológico de 6 casos. Revista do Instituto de Medicina Tropical de São Paulo 2:319-334, 1960.

20. Lopes ER, Chapadeiro E, Rocha A. Anatomia patológica do coração na forma indeterminada. In: Cançado JR, Chuster M (eds) Cardiopatia chagásica. Fundação Carlos Chagas, Belo Horizonte,
MG, p.33-40, 1985.

21. Lopes ER, Chapadeiro E, Tafuri WL, Prata AR. Doença de Chagas. In: Brasileiro Filho G, Pittela JEH, Pereira FEL, Bambirra EA, Barbosa AJA (eds) Bogliolo Patologia, Guanabara Koogan, Rio de Janeiro, p. 1103-1124, 1198-1199, 1994.

22. Matthews BR, Clark A. Neural control of the endocrine pancreas. Proceedings of the Nutrition Society 46:89-95, 1987.

23. Mott CB, Guarita DR, Sipahi AM, Bettarello A. Avaliação funcional do pâncreas exócrino em portadores de doença de Chagas crônica. Revista do Hospital das Clínicas da Faculdade de Medicina da Universidade de São Paulo 43:279-287, 1988.

24. Okumura M, Brito T, Silva LHP, Silva AC, Corrêa Neto A. The pathology of experimental Chagas disease in mice; I. Digestive tract changes, with a reference to necrotizing arteritis. Revista do Instituto de Medicina Tropical de São Paulo 2:17-28, 1960.

25. Oliveira LCM, Juliano Y, Novo NF, Neves MM. Blood glucose and insulin response to intravenous glucose by patients with chronic Chagas' disease and alcoholism. Brazilian Journal of Medical and Biological Research 26:1187-1190, 1993.

26. Pizzi TP. Localización pancreática predominante y fenómenos de esteatonecrosis en ratones experimentalmente infectados com Trypanosoma cruzi. Boletin de Informaciones Parasitarias Chilenas 8:11-13, 1953.

27. Reis LCF. Estudo sobre o metabolismo dos hidratos de carbono na moléstia de Chagas. I: A prova oral de tolerância à glicose. Revista da Associação Médica Brasileira 11:3-10, 1965.

28. Reis LCF, Vichi FL. Estudo sobre o metabolismo dos hidratos de carbono na moléstia de Chagas. II: A prova do glucagon. Revista da Associação Médica Brasileira 11:61-65, 1965.

29. Saldanha JC. Avaliação morfológica e morfométrica das ilhotas pancreáticas na fase crônica da doença de Chagas. Tese de Mestrado, Faculdade de Medicina do Triângulo Mineiro, Uberaba, MG, 1996.

30. Stagner JI, Samols E. Role of intrapancreatic ganglia in regulation of periodic insular secretions. American Journal of Physiology 248:E522-E530, 1985.

31. Tafuri WL, Raso P. Anatomia patológica. In: Raia AA (ed) Manifestações digestivas da moléstia de Chagas, Sarvier, São Paulo, p.61-79, 1983.

32. Vieira CB, Hadler WA. Estudo histológico da glândula parótida e do pâncreas no megaesôfago. Revista da Associação Médica Brasileira 7:89-96, 1961. 
33. Vieira CB, Meneghelli UG. Peculiaridades da hipoglicemia insulínica na forma crônica da moléstia de Chagas. I. Estudo clínico. Revista do Instituto de
Medicina Tropical de São Paulo 12:175-178, 1970.

34. Vieira CB, Soubihe NV, Ferriolli Filho F. Peculiaridades da hipoglicemia insulínica na forma 